DESY 07-070

May 24, 2007

\title{
Measurement of (anti)deuteron and (anti)proton production in DIS at HERA
}

\author{
ZEUS Collaboration
}

\begin{abstract}
The first observation of (anti)deuterons in deep inelastic scattering at HERA has been made with the ZEUS detector at a centre-of-mass energy of 300-318 GeV using an integrated luminosity of $120 \mathrm{pb}^{-1}$. The measurement was performed in the central rapidity region for transverse momentum per unit of mass in the range $0.3<p_{T} / M<0.7$. The particle rates have been extracted and interpreted in terms of the coalescence model. The (anti)deuteron production yield is smaller than the (anti)proton yield by approximately three orders of magnitude, consistent with the world measurements.
\end{abstract}




\section{The ZEUS Collaboration}

S. Chekanov ${ }^{1}$, M. Derrick, S. Magill, B. Musgrave, D. Nicholass ${ }^{2}$, J. Repond, R. Yoshida Argonne National Laboratory, Argonne, Illinois 60439-4815, USA ${ }^{n}$

M.C.K. Mattingly

Andrews University, Berrien Springs, Michigan 49104-0380, USA

M. Jechow, N. Pavel ${ }^{\dagger}$, A.G. Yagües Molina

Institut für Physik der Humboldt-Universität zu Berlin, Berlin, Germany

S. Antonelli, P. Antonioli, G. Bari, M. Basile, L. Bellagamba, M. Bindi, D. Boscherini, A. Bruni, G. Bruni, L. Cifarelli, F. Cindolo, A. Contin, M. Corradi, S. De Pasquale, G. Iacobucci, A. Margotti, R. Nania, A. Polini, G. Sartorelli, A. Zichichi University and INFN Bologna, Bologna, Italy $^{e}$

D. Bartsch, I. Brock, S. Goers ${ }^{3}$, H. Hartmann, E. Hilger, H.-P. Jakob, M. Jüngst, O.M. Kind ${ }^{4}$, A.E. Nuncio-Quiroz, E. Paul ${ }^{5}$, R. Renner ${ }^{6}$, U. Samson, V. Schönberg, R. Shehzadi, M. Wlasenko Physikalisches Institut der Universität Bonn, Bonn, Germany ${ }^{b}$

N.H. Brook, G.P. Heath, J.D. Morris, T. Namsoo

H.H. Wills Physics Laboratory, University of Bristol, Bristol, United Kingdom ${ }^{m}$

M. Capua, S. Fazio, A. Mastroberardino, M. Schioppa, G. Susinno, E. Tassi

Calabria University, Physics Department and INFN, Cosenza, Italy ${ }^{e}$

J.Y. $\mathrm{Kim}^{7}$, K.J. $\mathrm{Ma}^{8}$

Chonnam National University, Kwangju, South Korea ${ }^{g}$

Z.A. Ibrahim, B. Kamaluddin, W.A.T. Wan Abdullah

Jabatan Fizik, Universiti Malaya, 50603 Kuala Lumpur, Malaysia ${ }^{r}$

Y. Ning, Z. Ren, F. Sciulli

Nevis Laboratories, Columbia University, Irvington on Hudson, New York 10027o

J. Chwastowski, A. Eskreys, J. Figiel, A. Galas, M. Gil, K. Olkiewicz, P. Stopa, L. Zawiejski

The Henryk Niewodniczanski Institute of Nuclear Physics, Polish Academy of Sciences, Cracow, Poland ${ }^{i}$

L. Adamczyk, T. Bołd, I. Grabowska-Bołd, D. Kisielewska, J. Łukasik, M. Przybycień, L. Suszycki

Faculty of Physics and Applied Computer Science, AGH-University of Science and Technology, Cracow, Poland ${ }^{p}$

A. Kotański ${ }^{9}$, W. Słomiński ${ }^{10}$

Department of Physics, Jagellonian University, Cracow, Poland 
V. Adler ${ }^{11}$, U. Behrens, I. Bloch, C. Blohm, A. Bonato, K. Borras, R. Ciesielski, N. Coppola, A. Dossanov, V. Drugakov, J. Fourletova, A. Geiser, D. Gladkov, P. Göttlicher ${ }^{12}$, J. Grebenyuk, I. Gregor, T. Haas, W. Hain, C. Horn ${ }^{13}$, A. Hüttmann, B. Kahle, I.I. Katkov, U. Klein ${ }^{14}$, U. Kötz, H. Kowalski, E. Lobodzinska, B. Löhr, R. Mankel, I.-A. MelzerPellmann, S. Miglioranzi, A. Montanari, D. Notz, L. Rinaldi, P. Roloff, I. Rubinsky, R. Santamarta, U. Schneekloth, A. Spiridonov ${ }^{15}$, H. Stadie, D. Szuba ${ }^{16}$, J. Szuba ${ }^{17}$,

T. Theedt, G. Wolf, K. Wrona, C. Youngman, W. Zeuner

Deutsches Elektronen-Synchrotron DESY, Hamburg, Germany

W. Lohmann, S. Schlenstedt

Deutsches Elektronen-Synchrotron DESY, Zeuthen, Germany

G. Barbagli, E. Gallo, P. G. Pelfer

University and INFN, Florence, Italy ${ }^{e}$

A. Bamberger, D. Dobur, F. Karstens, N.N. Vlasov ${ }^{18}$

Fakultät für Physik der Universität Freiburg i.Br., Freiburg i.Br., Germany ${ }^{b}$

P.J. Bussey, A.T. Doyle, W. Dunne, J. Ferrando, M. Forrest, D.H. Saxon, I.O. Skillicorn

Department of Physics and Astronomy, University of Glasgow, Glasgow, United Kingdom $^{m}$

I. Gialas ${ }^{19}$, K. Papageorgiu

Department of Engineering in Management and Finance, Univ. of Aegean, Greece

T. Gosau, U. Holm, R. Klanner, E. Lohrmann, H. Salehi, P. Schleper, T. Schörner-Sadenius, J. Sztuk, K. Wichmann, K. Wick

Hamburg University, Institute of Exp. Physics, Hamburg, Germany ${ }^{b}$

C. Foudas, C. Fry, K.R. Long, A.D. Tapper

Imperial College London, High Energy Nuclear Physics Group, London, United Kingdom $^{m}$

M. Kataoka ${ }^{20}$, T. Matsumoto, K. Nagano, K. Tokushuku², S. Yamada, Y. Yamazaki Institute of Particle and Nuclear Studies, KEK, Tsukuba, Japan ${ }^{f}$

A.N. Barakbaev, E.G. Boos, N.S. Pokrovskiy, B.O. Zhautykov

Institute of Physics and Technology of Ministry of Education and Science of Kazakhstan, Almaty, Kazakhstan

V. Aushev ${ }^{1}$

Institute for Nuclear Research, National Academy of Sciences, Kiev and Kiev National University, Kiev, Ukraine

D. Son

Kyungpook National University, Center for High Energy Physics, Daegu, South Korea ${ }^{g}$

J. de Favereau, K. Piotrzkowski

Institut de Physique Nucléaire, Université Catholique de Louvain, Louvain-la-Neuve, Belgium $^{q}$ 
F. Barreiro, C. Glasman²2, M. Jimenez, L. Labarga, J. del Peso, E. Ron, M. Soares,

J. Terrón, M. Zambrana

Departamento de Física Teórica, Universidad Autónoma de Madrid, Madrid, Spain ${ }^{l}$

F. Corriveau, C. Liu, R. Walsh, C. Zhou

Department of Physics, McGill University, Montréal, Québec, Canada H3A 2T8 ${ }^{a}$

T. Tsurugai

Meiji Gakuin University, Faculty of General Education, Yokohama, Japan ${ }^{f}$

A. Antonov, B.A. Dolgoshein, V. Sosnovtsev, A. Stifutkin, S. Suchkov

Moscow Engineering Physics Institute, Moscow, Russia ${ }^{j}$

R.K. Dementiev, P.F. Ermolov, L.K. Gladilin, L.A. Khein, I.A. Korzhavina, V.A. Kuzmin,

B.B. Levchenko ${ }^{23}$, O.Yu. Lukina, A.S. Proskuryakov, L.M. Shcheglova, D.S. Zotkin, S.A. Zotkin

Moscow State University, Institute of Nuclear Physics, Moscow, Russia ${ }^{k}$

I. Abt, C. Büttner, A. Caldwell, D. Kollar, W.B. Schmidke, J. Sutiak

Max-Planck-Institut für Physik, München, Germany

G. Grigorescu, A. Keramidas, E. Koffeman, P. Kooijman, A. Pellegrino, H. Tiecke, M. Vázquez ${ }^{20}$, L. Wiggers

NIKHEF and University of Amsterdam, Amsterdam, Netherlands ${ }^{h}$

N. Brümmer, B. Bylsma, L.S. Durkin, A. Lee, T.Y. Ling

Physics Department, Ohio State University, Columbus, Ohio 43210 ${ }^{n}$

P.D. Allfrey, M.A. Bell, A.M. Cooper-Sarkar, A. Cottrell, R.C.E. Devenish, B. Foster, K. Korcsak-Gorzo, S. Patel, V. Roberfroid ${ }^{24}$, A. Robertson, P.B. Straub, C. UribeEstrada, R. Walczak

Department of Physics, University of Oxford, Oxford United Kingdom ${ }^{m}$

P. Bellan, A. Bertolin, R. Brugnera, R. Carlin, F. Dal Corso, S. Dusini, A. Garfagnini,

S. Limentani, A. Longhin, L. Stanco, M. Turcato

Dipartimento di Fisica dell' Università and INFN, Padova, Italy ${ }^{e}$

B.Y. Oh, A. Raval, J. Ukleja ${ }^{25}$, J.J. Whitmore ${ }^{26}$

Department of Physics, Pennsylvania State University, University Park, Pennsylvania $16802^{\circ}$

Y. Iga

Polytechnic University, Sagamihara, Japan ${ }^{f}$

G. D'Agostini, G. Marini, A. Nigro

Dipartimento di Fisica, Università 'La Sapienza' and INFN, Rome, Italy ${ }^{e}$

J.E. Cole, J.C. Hart

Rutherford Appleton Laboratory, Chilton, Didcot, Oxon, United Kingdom ${ }^{m}$ 
H. Abramowicz ${ }^{27}$, A. Gabareen, R. Ingbir, S. Kananov, A. Levy

Raymond and Beverly Sackler Faculty of Exact Sciences, School of Physics, Tel-Aviv University, Tel-Aviv, Israel ${ }^{d}$

M. Kuze, J. Maeda

Department of Physics, Tokyo Institute of Technology, Tokyo, Japan ${ }^{f}$

R. Hori, S. Kagawa ${ }^{28}$, N. Okazaki, S. Shimizu, T. Tawara

Department of Physics, University of Tokyo, Tokyo, Japan ${ }^{f}$

R. Hamatsu, H. Kaji2 ${ }^{29}$, S. Kitamura ${ }^{30}$, O. Ota, Y.D. Ri

Tokyo Metropolitan University, Department of Physics, Tokyo, Japan ${ }^{f}$

M.I. Ferrero, V. Monaco, R. Sacchi, A. Solano

Università di Torino and INFN, Torino, Italy ${ }^{e}$

M. Arneodo, M. Ruspa

Università del Piemonte Orientale, Novara, and INFN, Torino, Italy ${ }^{e}$

S. Fourletov, J.F. Martin

Department of Physics, University of Toronto, Toronto, Ontario, Canada M5S 1A7 a

S.K. Boutle ${ }^{19}$, J.M. Butterworth, C. Gwenlan ${ }^{31}$, T.W. Jones, J.H. Loizides, M.R. Sutton ${ }^{31}$, M. Wing

Physics and Astronomy Department, University College London, London, United Kingdom $^{m}$

B. Brzozowska, J. Ciborowski ${ }^{32}$, G. Grzelak, P. Kulinski, P. Łużniak ${ }^{33}$, J. Malka ${ }^{33}$, R.J. Nowak, J.M. Pawlak, T. Tymieniecka, A. Ukleja, A.F. Żarnecki

Warsaw University, Institute of Experimental Physics, Warsaw, Poland

M. Adamus, P. Plucinski ${ }^{34}$

Institute for Nuclear Studies, Warsaw, Poland

Y. Eisenberg, I. Giller, D. Hochman, U. Karshon, M. Rosin

Department of Particle Physics, Weizmann Institute, Rehovot, Israel ${ }^{c}$

E. Brownson, T. Danielson, A. Everett, D. Kçira, D.D. Reeder ${ }^{5}$, P. Ryan, A.A. Savin, W.H. Smith, H. Wolfe

Department of Physics, University of Wisconsin, Madison, Wisconsin 53706, USA ${ }^{n}$

S. Bhadra, C.D. Catterall, Y. Cui, G. Hartner, S. Menary, U. Noor, J. Standage, J. Whyte Department of Physics, York University, Ontario, Canada M3J 1Pз ${ }^{a}$ 
${ }^{1}$ supported by DESY, Germany

2 also affiliated with University College London, UK

${ }^{3}$ now with TÜV Nord, Germany

${ }^{4}$ now at Humboldt University, Berlin, Germany

${ }^{5}$ retired

${ }^{6}$ self-employed

${ }^{7}$ supported by Chonnam National University in 2005

${ }^{8}$ supported by a scholarship of the World Laboratory Björn Wiik Research Project

9 supported by the research grant no. 1 P03B 04529 (2005-2008)

10 This work was supported in part by the Marie Curie Actions Transfer of Knowledge project COCOS (contract MTKD-CT-2004-517186)

11 now at Univ. Libre de Bruxelles, Belgium

12 now at DESY group FEB, Hamburg, Germany

13 now at Stanford Linear Accelerator Center, Stanford, USA

14 now at University of Liverpool, UK

15 also at Institut of Theoretical and Experimental Physics, Moscow, Russia

16 also at INP, Cracow, Poland

17 on leave of absence from FPACS, AGH-UST, Cracow, Poland

18 partly supported by Moscow State University, Russia

19 also affiliated with DESY

${ }^{20}$ now at CERN, Geneva, Switzerland

21 also at University of Tokyo, Japan

${ }^{22}$ Ramón y Cajal Fellow

23 partly supported by Russian Foundation for Basic Research grant no. 05-02-39028NSFC-a

${ }^{24}$ EU Marie Curie Fellow

${ }^{25}$ partially supported by Warsaw University, Poland

26 This material was based on work supported by the National Science Foundation, while working at the Foundation.

27 also at Max Planck Institute, Munich, Germany, Alexander von Humboldt Research Award

28 now at KEK, Tsukuba, Japan

${ }^{29}$ now at Nagoya University, Japan

${ }^{30}$ Department of Radiological Science

${ }^{31}$ PPARC Advanced fellow

32 also at Łódź University, Poland

33 Łódź University, Poland

${ }^{34}$ supported by the Polish Ministry for Education and Science grant no. 1 P03B 14129

$\dagger$ deceased 
a supported by the Natural Sciences and Engineering Research Council of Canada (NSERC)

$b$ supported by the German Federal Ministry for Education and Research (BMBF), under contract numbers HZ1GUA 2, HZ1GUB 0, HZ1PDA 5, HZ1VFA 5

c supported in part by the MINERVA Gesellschaft für Forschung GmbH, the Israel Science Foundation (grant no. 293/02-11.2) and the U.S.-Israel Binational Science Foundation

$d$ supported by the German-Israeli Foundation and the Israel Science Foundation

$e$ supported by the Italian National Institute for Nuclear Physics (INFN)

$f$ supported by the Japanese Ministry of Education, Culture, Sports, Science and Technology (MEXT) and its grants for Scientific Research

$g$ supported by the Korean Ministry of Education and Korea Science and Engineering Foundation

$h$ supported by the Netherlands Foundation for Research on Matter (FOM)

$i$ supported by the Polish State Committee for Scientific Research, grant no. 620/E-77/SPB/DESY/P-03/DZ 117/2003-2005 and grant no. 1P03B07427/2004-2006

j partially supported by the German Federal Ministry for Education and Research $(\mathrm{BMBF})$

$k \quad$ supported by RF Presidential grant N 8122.2006.2 for the leading scientific schools and by the Russian Ministry of Education and Science through its grant Research on High Energy Physics

$l$ supported by the Spanish Ministry of Education and Science through funds provided by CICYT

$m$ supported by the Particle Physics and Astronomy Research Council, UK

$n$ supported by the US Department of Energy

o supported by the US National Science Foundation. Any opinion, findings and conclusions or recommendations expressed in this material are those of the authors and do not necessarily reflect the views of the National Science Foundation.

$p \quad$ supported by the Polish Ministry of Science and Higher Education as a scientific project (2006-2008)

$q$ supported by FNRS and its associated funds (IISN and FRIA) and by an Inter-University Attraction Poles Programme subsidised by the Belgian Federal Science Policy Office

$r$ supported by the Malaysian Ministry of Science, Technology and Innovation/Akademi Sains Malaysia grant SAGA 66-02-03-0048 


\section{Introduction}

Light stable nuclei, such as deuterons $(d)$ and tritons $(t)$, are loosely bound states whose production mechanism in high-energy collisions is poorly understood. Most measurements of light stable nuclei have been performed for antideuterons $(\bar{d})$. A selection of $d$ from primary interactions is more difficult as it requires separation of such states from particles produced by interactions of colliding beams with residual gas in the beam pipe and by secondary interactions in detector material. The first observation of $\bar{d}$ [1] was followed by a number of experiments on antideuteron production. The production rate of $\bar{d}$ in $e^{+} e^{-} \rightarrow q \bar{q}$ collisions $[2-5]$ is significantly lower than that measured in $\Upsilon(1 S)$ and $\Upsilon(2 S)$ decays $[2,5]$. The $\bar{d}$ rate in $e^{+} e^{-} \rightarrow q \bar{q}$ is also lower than that in proton-nucleus $(p A)[6,7]$, proton-proton $(p p)$ [8] and photon-proton $(\gamma p)$ collisions at HERA [9], but higher than that in nucleus-nucleus collisions [10,11]. For heavy-ion collisions, the coalescence model [12] was proposed to explain the production of $d(\bar{d})$.

This paper presents the results of the first measurement of $d$ and $\bar{d}$ in the central rapidity region of deep inelastic ep scattering (DIS). The analysis was performed for exchanged photon virtuality, $Q^{2}$, above $1 \mathrm{GeV}^{2}$.

\section{Coalescence model for (anti)deuteron formation}

According to the coalescence model [12] developed for heavy-ion collisions, the production rate of $d$ is determined by the overlap between the wave-function of a proton $(p)$ and a neutron $(n)$ with the wave-function of a $d$. In this case, the $d$ cross section is the product of single-particle cross sections for protons and neutrons, with a coefficient of proportionality reflecting the spatial size of the fragmentation region emitting the particles. The same approach applies for $\bar{d}$ production. This model was also used to describe $d(\bar{d})$ production in $p p$ [8], $\gamma p[9]$ and $e^{+} e^{-}[2,4]$ interactions.

Assuming that all baryons are uncorrelated and the invariant differential cross section for neutrons is equal to that for protons, the invariant differential cross section for deuteron formation can be parameterised as

$$
\frac{E_{d}}{\sigma_{t o t}} \frac{\mathrm{d}^{3} \sigma_{d}}{\mathrm{~d} p_{d}^{3}}=B_{2}\left(\frac{E_{p}}{\sigma_{t o t}} \frac{\mathrm{d}^{3} \sigma_{p}}{\mathrm{~d} p_{p}^{3}}\right)^{2}
$$

where $E_{d(p)}$ and $\sigma_{d(p)}$ are the energy and the production cross section of the $d(p)$, respectively, $p_{d}\left(p_{p}\right)$ is the momentum of the $d(p)$ and $\sigma_{\text {tot }}$ is the total ep cross section for the considered kinematic range. The coalescence parameter, $B_{2}$, is inversely proportional to the volume of the fragmentation region emitting the particles. The same relation holds for $\bar{d}$ and $\bar{p}$. If $B_{2}$ is the same for particles and antiparticles, then the production ratio 
$\bar{d} / d$ is equal to $(\bar{p} / p)^{2}$. The coalescence parameter can be obtained from

$$
B_{2}=\left(\frac{E_{d}}{\sigma_{t o t}} \frac{\mathrm{d}^{3} \sigma_{d}}{\mathrm{~d} p_{d}^{3}}\right)\left(\frac{E_{p}}{\sigma_{t o t}} \frac{\mathrm{d}^{3} \sigma_{p}}{\mathrm{~d} p_{p}^{3}}\right)^{-2}=M_{p}^{4} M_{d}^{-2} R^{2}(d / p)\left(\frac{\gamma_{d}}{\sigma_{t o t}} \frac{\mathrm{d}^{3} \sigma_{d}}{\mathrm{~d}\left(p_{d} / M_{d}\right)^{3}}\right)^{-1}
$$

where $M_{d(p)}$ is the mass of the $d(p), \gamma_{d}=E_{d} / M_{d}, R(d / p)$ is the ratio of the number of $d$ to $p$ expressed as a function of $p_{T} / M_{d(p)}$, with $p_{T}$ being the transverse momentum [9].

\section{Experimental set-up}

A detailed description of the ZEUS detector can be found elsewhere [13]. A brief outline of the components that are most relevant for this analysis is given below.

Charged particles are tracked in the central tracking detector (CTD) [14], which operates in a magnetic field of $1.43 \mathrm{~T}$ provided by a thin superconducting solenoid. The CTD consists of 72 cylindrical drift chamber layers, organised in nine superlayers covering the polar-angl 1 region $15^{\circ}<\theta<164^{\circ}$. The transverse-momentum resolution for full-length tracks is $\sigma\left(p_{T}\right) / p_{T}=0.0058 p_{T} \oplus 0.0065 \oplus 0.0014 / p_{T}$, with $p_{T}$ in $\mathrm{GeV}$. To estimate the ionisation energy loss per unit length, $d E / d x$, of particles in the CTD [15], the truncated mean of the anode-wire pulse heights was calculated, which removes the lowest $10 \%$ and at least the highest $30 \%$ depending on the number of saturated hits. The measured $d E / d x$ values were corrected by normalising to the average $d E / d x$ for tracks around the region of minimum ionisation for pions with momentum $p$ satisfying $0.3<p<0.4 \mathrm{GeV}$. Henceforth, $d E / d x$ is quoted in units of minimum ionising particles (mips). The resolution of the $d E / d x$ measurement for full-length tracks is about $9 \%$.

The high-resolution uranium-scintillator calorimeter (CAL) [16] consists of three parts: the forward (FCAL), the barrel (BCAL) and the rear (RCAL) calorimeters. Each part is subdivided transversely into towers and longitudinally into one electromagnetic section (EMC) and either one (in RCAL) or two (in BCAL and FCAL) hadronic sections (HAC). The smallest subdivision of the calorimeter is called a cell. The CAL energy resolutions, as measured under test-beam conditions, are $\sigma(E) / E=0.18 / \sqrt{E}$ for electrons and $\sigma(E) / E=0.35 / \sqrt{E}$ for hadrons, with $E$ in $\mathrm{GeV}$. A presampler [17] mounted in front of the calorimeter and a scintillator-strip detector (SRTD) [18] were used to correct the energy of the scattered electron 2. The position of electrons scattered close to the electron beam direction is determined by the SRTD detector.

The inactive material between the interaction region and the CTD, relevant for this analysis, consists of the central beam pipe made of aluminum with $1.5 \mathrm{~mm}$ wall thickness

\footnotetext{
${ }^{1}$ The ZEUS coordinate system is a right-handed Cartesian system, with the $Z$ axis pointing in the proton beam direction, referred to as the "forward direction", and the $X$ axis pointing left towards the centre of HERA. The coordinate origin is at the nominal interaction point.

${ }^{2}$ Henceforth the term electron is used to refer both to electrons and positrons.
} 
and the inner diameter of $135 \mathrm{~mm}$. The CTD inner wall with a diameter of $324 \mathrm{~mm}$ consists of two aluminum skins, each $0.7 \mathrm{~mm}$ thick, separated by a $8.6 \mathrm{~mm}$ gap filled with polyurethane foam with a nominal density of $0.05 \mathrm{~g} / \mathrm{cm}^{3}$.

The luminosity was measured using the bremsstrahlung process $e p \rightarrow e p \gamma$ with the luminosity monitor [19], a lead-scintillator calorimeter placed in the HERA tunnel at $Z=-107 \mathrm{~m}$.

\section{Monte Carlo simulation}

To study the detector response, the ARIADne 4.12 Monte Carlo (MC) model [20] for the description of inclusive DIS events was used. The ARIADNE program uses the Lund string model [21] for hadronisation, as implemented in Pyтніа 6.2 [22-24]. In its original version, this $\mathrm{MC}$ does not include a mechanism for the production of $d$ or other light stable nuclei. To determine reconstruction efficiencies, a second ARIADNE sample was generated in which $d$ 's were included at the generator level by combining $p$ and $n$ with similar momenta.

The ARIADne events were passed through a full simulation of the detector using the Geant 3.13 [25] program. The Geant simulation uses the Gheisha model [26] to simulate hadronic interactions in the material. The GEANT program cannot be used for $\bar{d}$ as this particle is not included in the particle table.

\section{Event sample}

\subsection{DIS event selection}

The data sample corresponds to an integrated luminosity of $120.3 \mathrm{pb}^{-1}$ taken between 1996 and 2000 with the ZEUS detector at HERA. This sample consists of $38.6 \mathrm{pb}^{-1}$ of $e^{+} p$ data taken at a centre-of-mass energy of $300 \mathrm{GeV}, 65.0 \mathrm{pb}^{-1}$ taken at $318 \mathrm{GeV}$ and $16.7 \mathrm{pb}^{-1}$ of $e^{-} p$ data taken at $318 \mathrm{GeV}$.

The search was performed using DIS events with exchanged-photon virtuality $Q^{2}>$ $1 \mathrm{GeV}^{2}$. The event selection was similar to that used in a previous ZEUS publication [27]. A three-level trigger [13] was used to select events online. At the third-level trigger, an electron with an energy greater than $4 \mathrm{GeV}$ was required. Data below $Q^{2} \approx 20 \mathrm{GeV}^{2}$ were prescaled to reduce trigger rates.

The Bjorken scaling variable, $x_{\mathrm{Bj}}$, and $Q^{2}$ were reconstructed using the electron method (denoted by the subscript $e$ ), which uses measurements of the energy and angle of the scattered electron. The scattered-electron candidate was identified from the pattern of energy deposits in the CAL [28]. In addition, the inelasticity was reconstructed using the Jacquet-Blondel method [29], $y_{\mathrm{JB}}$, or the electron method, $y_{e}$. 
For the final DIS sample, the following requirements were imposed:

- $Q_{e}^{2}>1 \mathrm{GeV}^{2}$;

- the impact point of the scattered electron on the RCAL outside the $(X, Y)$ region $( \pm 12, \pm 6) \mathrm{cm}$ centred on the beamline;

- $E_{e^{\prime}}>8.5 \mathrm{GeV}$, where $E_{e^{\prime}}$ is the energy of the scattered electron measured in the CAL and corrected for energy losses;

- $35<\delta<65 \mathrm{GeV}$, where $\delta=\sum E_{i}\left(1-\cos \theta_{i}\right), E_{i}$ is the energy of the $i$-th calorimeter cell, $\theta_{i}$ is its polar angle and the sum runs over all cells;

- $y_{e}<0.95$ and $y_{\mathrm{JB}}>0.01$

- at least three tracks fitted to the primary vertex to ensure a good reconstruction of the primary vertex and to reduce contributions from non-ep events;

- $\left|Z_{\mathrm{vtx}}\right|<40 \mathrm{~cm}$ and $\sqrt{X_{\mathrm{vtx}}^{2}+Y_{\mathrm{vtx}}^{2}}<1 \mathrm{~cm}$, where $Z_{\mathrm{vtx}}, X_{\mathrm{vtx}}$ and $Y_{\mathrm{vtx}}$ are the coordinates of the vertex position determined from the tracks.

The average $Q^{2}$ of the selected sample was about $10 \mathrm{GeV}^{2}$.

\subsection{Track selection and the $d E / d x$ measurement}

The present analysis is based on charged tracks measured in the CTD. The tracks were required to have:

- at least $40 \mathrm{CTD}$ hits, with at least 8 of them for the $d E / d x$ measurement;

- the transverse momentum $p_{T} \geq 0.15 \mathrm{GeV}$.

These cuts selected a region where the CTD track acceptance, as well as the resolutions in momentum and the $d E / d x$, were high.

To identify particles originating from $e p$ collisions, the following additional variables were reconstructed for each track:

- the distance, $\Delta Z$, of the $Z$-component of the track helix to $Z_{\mathrm{vtx}}$;

- the distance of closest approach $(D C A)$ of the track to the beam-spot location in the transverse plane. The beam-spot position is determined from the average primaryvertex distributions in $X$ and $Y$ for each data-taking period. The $D C A$ is assigned a positive (negative) value if the beam spot lies left (right) of the particle path.

Figure 1 shows the $d E / d x$ distribution as a function of the track momentum for positive and negative tracks. The events were selected by requiring at least one track with $d E / d x>$ 2.5 mips. To reduce the fraction of tracks coming from non-ep collisions, the tracks were required to have $|\Delta Z|<1 \mathrm{~cm}$ and $|D C A|<0.5 \mathrm{~cm}$. After such a selection, clear bands corresponding to charged kaons, protons and deuterons were observed. The requirement $d E / d x>2.5$ mips enhances the fraction of events with at least one particle with a mass 
larger than the pion mass and leads to the discontinuity near $d E / d x=2.5$ mips seen in Figure 1. The lines show the most probable energy loss calculated from the Bethe-Bloch formula [30]. The $d E / d x$ bands for $K^{-}$and $\bar{p}$ are slightly shifted with respect to the Bethe-Bloch expectations due to the geometrical structure of the CTD drift cells which leads to a different response to negative and positive tracks.

Figure 2 shows the reconstructed masses, $M$, for different particle species. The masses were calculated from the measured track momentum and energy loss using the BetheBloch formula. The mass distributions were fitted with asymmetrid 3 Gaussian functions. The relative width obtained was 11\% (7\%) for the left (right) part of the function.

The number of $p(\bar{p})$ candidates in the mass region $0.7(0.6)<M<1.5 \mathrm{GeV}$ was $1.61 \times 10^{5}$ $\left(1.66 \times 10^{5}\right)$. Due to a shift in the $d E / d x$ for negative tracks, the lower mass cut for $\bar{p}$ was at $0.6 \mathrm{GeV}$. The numbers of $d$ and $\bar{d}$ in the mass window $1.5<M<2.5 \mathrm{GeV}$ were 309 and 62 , respectively. The number of $p$ migrating to the $d$ mass region was estimated to be less than $1 \%$ of the total number of $d$ candidates. A similar estimate was obtained for antiparticles. A small number of triton candidates was observed in the mass window $2.5<M<3.5 \mathrm{GeV}$. However, due to low statistics, it was difficult to establish a peak inside this mass window, therefore, no conclusive statement on the origin of the tracks in the region $2.5<M<3.5 \mathrm{GeV}$ was possible.

The observed $p(\bar{p})$ and $d(\bar{d})$ candidates were required to be in the central rapidity region, $|y|<0.4$, and to have $0.3<p_{T} / M<0.7$. This determines the kinematic range used for the cross-section calculations.

\subsection{Identification of particles produced in $e p$ collisions}

The observed $p(\bar{p})$ and $d(\bar{d})$ candidates selected after the $d E / d x$ mass cuts can originate from secondary interactions in the inactive material between the interaction point and the central tracking detector.

In order to select $p(\bar{p})$ and $d(\bar{d})$ originating from ep collisions, both $D C A$ and $\Delta Z$ cuts were removed and a statistical background subtraction based on the $D C A$ distribution was performed. The $\Delta Z$ distributions for $p(\bar{p})$ and $d(\bar{d})$ after the mass cuts are shown in Figure 3. Clear peaks at $\Delta Z=0$ are observed. To optimize the signal-over-background ratio for the $D C A$ distribution, all candidates were selected using the $|\Delta Z|<2(1) \mathrm{cm}$ restriction for $p, \bar{p}(d, \bar{d})$.

Figure 4 shows the $D C A$ distributions for $p(\bar{p})$ and $d(\bar{d})$ candidates. The distributions show peaks at zero due to tracks originating from the primary vertex. The number of particles originating from primary ep collisions was determined using the side-band background subtraction. A linear fit to the $D C A$ distribution on either side of the peak region in the range $2<|D C A|<4 \mathrm{~cm}$ was performed. Then, the expected number of

\footnotetext{
${ }^{3}$ An asymmetric Gaussian has different widths for the left and right parts of the function.
} 
background events in the signal region of $|D C A|<1.5(0.5) \mathrm{cm}$ for $p, \bar{p}(d, \bar{d})$ candidates was subtracted.

The number of $p(\bar{p})$ obtained after the $D C A$ side-band background subtraction was $1.52 \times$ $10^{5}\left(1.62 \times 10^{5}\right)$. The numbers of $d$ and $\bar{d}$ particles were $177 \pm 17$ and $53 \pm 7$, respectively. The difference in the observed numbers of $p$ and $\bar{p}$ can be explained by different $d E / d x$ efficiencies and the mass cuts for positive and negative tracks. Such a difference in the efficiencies for particles and antiparticles cannot explain the difference in the observed numbers of $d$ and $\bar{d}$.

Figure 5 shows the distributions for several DIS kinematic variables: $Q_{e}^{2}, x_{e}, E_{e^{\prime}}$ and $\delta$. In addition, rapidity $(y)$ distributions for the selected candidates are shown. The numbers of $p(\bar{p})$ and $d(\bar{d})$ candidates were calculated in each bin from the $D C A$ distributions after the side-band background subtraction. The distributions for $d$ are consistent with those for $p$ and $\bar{p}$, while the $\bar{d}$ sample shows some deviations for the $E_{e^{\prime}}$ variable and, consequently, for the $\delta$ variable.

\section{$6 \quad$ Studies of background processes}

The following two background sources for heavy stable charged particles were considered:

- interactions of the proton (or electron) beam with residual gas in the beam pipe, termed beam-gas interactions;

- secondary interactions of particles in inactive material between the interaction point and the central tracking detector.

\subsection{Beam-gas interactions}

The contribution from proton-gas interactions is significantly reduced after the ZEUS three-level trigger which requires a scattered electron in the CAL. In addition, the requirement to accept only events with more than three tracks fitted to the primary vertex significantly diminishes the contribution from both electron-gas and proton-gas events. The remaining fraction of beam-gas interactions can be assessed by studying the $Z_{\mathrm{vtx}}$ distribution.

Figure 6 shows the $Z_{\mathrm{vtx}}$ distributions for events with at least one $p(\bar{p})$ or $d(\bar{d})$ candidate. The distributions were reconstructed in the signal region $|\Delta Z|<2(1) \mathrm{cm}$ and $|D C A|<$ $1.5(0.5) \mathrm{cm}$ for $p, \bar{p}(d, \bar{d})$ candidates without the background subtraction. Figure 6 shows that there is essentially no beam-gas background for $\bar{d}$ events. A small background for $d$ at positive $Z_{\mathrm{vtx}}$ is expected from the DIS MC generated for inclusive DIS events in which $d$ 's are solely produced by secondary interactions in the material in front of the CTD. This background is expected to have a flat DCA and, therefore, is subtracted by the procedure described in Section 5.3 
The $Z_{\mathrm{vtx}}$ distributions were fitted using a Gaussian function with a first-order polynomial for the background description. The extracted Gaussian widths are fully consistent with those obtained for inclusive DIS events without the $d$ preselection.

To further study the $Z_{\mathrm{vtx}}$ distribution, a special event selection was performed for noncolliding electron and proton bunches. Since the requirement to detect an electron with energy $E_{e^{\prime}} \geq 8.5 \mathrm{GeV}$ significantly reduces the rate of such background events, this requirement was not applied. All other tracking cuts were the same as in the $d(\bar{d})$ selection. The requirement to accept events with at least three tracks fitted to the primary vertex rejects most of the beam-gas events $(\sim 95 \%$ from the total number of the triggered events). As expected, the remaining events show clear peaks at zero for the $\triangle Z$ and $D C A$ distributions, but the reconstructed $Z_{\mathrm{vtx}}$ distribution did not show a peak at zero.

The enhancement at large $Z_{\mathrm{vtx}}$ for $d$, which was found to be consistent with that originating from secondary interactions, could partially be due to electron-gas interactions. If one assumes that the background seen in Fig. 6(b) is due to non-ep interactions, then the contribution from beam-gas interactions does not exceed $17 \%$ of the total number of events with a deuteron.

\subsection{Secondary interactions on inactive material}

A pure sample of DIS events will still contain deuterons produced by secondary interactions of particles in material. The aim of the side-band background subtraction discussed in Sect.5.3 was to remove such a background contribution, assuming that the background processes do not create a residual peak at $\triangle Z=0$ and $D C A=0$. Several checks of this assumption are discussed below.

The $D C A$ and $\triangle Z$ distributions were investigated using a $\mathrm{MC}$ simulation of inclusive DIS events without $d(\bar{d})$ production at the generator level. Deuterons from secondary interactions were selected as for the data. The reconstructed $D C A$ and $\triangle Z$ for $d$ did not show a peak at zero. A more detailed study of the $D C A$ and $\triangle Z$ distributions was possible for $p$ not originating from an ep collision at the $\mathrm{MC}$ generator level, since in this case the available MC statistics is significantly higher than for the $d$ case. After the track-quality cuts, no peak at zero was observed in the $D C A$ and $\Delta Z$ distributions.

If a deuteron is produced by secondary interactions of the particles from the DIS event in the surrounding matter, the secondary $d$ will not point precisely back to the interaction point, and both $D C A$ and $\Delta Z$ distributions will be wider than in case of $\bar{d}$ and $\bar{p}$. Therefore, the $D C A$ and $\triangle Z$ distributions were fitted with double-Gaussian distributions to establish the width of the distributions. It was found that the observed deuteron $D C A$ and $\Delta Z$ widths were consistent with the corresponding widths for $p$ and $\bar{p}$.

One possible source for $d$ is the reaction $N+N \rightarrow d+\pi$, where one of the nucleons $N$ originates from ep collision, while the other one originates from the detector material in front of the CTD. For low initial nucleon momenta, the $D C A$ of the $d$ track is in general 
large and it does not form an important background; at high initial nucleon momenta however, the $D C A$ can become small enough that misidentification could become important 4 . Since the processes $N+N \rightarrow d+\pi$ can lead to an additional charged pion, this source of background deuterons can be studied by comparing the average charged multiplicity of tracks for $d$ and $\bar{d}$ events. In addition, the distance of closest approach, $D C A 12$, between the $d$ track and other non-primary tracks in the same event should have an enhancement at zero. The study indicated that the average number of tracks for $d$ events is smaller than that for $\bar{d}$ events. The rejection of events with $|D C A 12|<2 \mathrm{~cm}$ did not lead to a statistically significant reduction in the number of the observed $d$ events.

Secondary deuterons may also be produced in pickup $(p+n \rightarrow d)$ reactions by primary $p(n)$ interacting in the surrounding material. These deuterons, peaking in the direction of the primary $p(n)$, point approximately to the interaction point and are therefore a potentially dangerous source of background. Experimental data on the pickup reactions at the relevant energy are scarce and therefore only a rough estimate of the size of this background is possible. From the extrapolation of data on $\mathrm{Sm}^{154}$ [31] and $C[32,33]$ targets using the K. Kikuchi theory [34] to allow for the change of material, the estimated $d$ background from the pickup reaction was in the range $1-10 \%$ of the total number of observed $d$ events, depending on the extrapolation input.

The angular distributions of $d$ from pickup reactions have also been investigated in several experiments $[32,35,36]$ for various targets and for a range of $p / M$ similar to the present analysis. In all cases, the angular distribution of $d$ observed in these experiments would lead to a much wider $D C A$ than that shown in Figure 4 (b).

\section{$7 \quad$ Detector corrections}

In this analysis, all measurements are based on event ratios, therefore, the detector corrections due to DIS event selection and trigger efficiency were found to be small and thus are not discussed here. The detector corrections for the tracking efficiency and the efficiency of the $d E / d x$ cuts are described below.

\subsection{Tracking efficiency}

The efficiency due to the track reconstruction, $\varepsilon$, was estimated separately for $p(\bar{p})$ and $d$ using the ARIADnE MC model (with $d$ included at the generator level). The obtained efficiencies are about 0.95 for $p$ and $d$ and 0.90 for $\bar{p}$.

The method cannot be applied to $\bar{d}$ which are not treated in the GEANT simulation. Therefore, the tracking efficiency for $\bar{d}$ was modelled as $\varepsilon(\bar{d})=\varepsilon(d) \varepsilon(\bar{p}) / \varepsilon(p)$. In the expression above, the hit reconstruction efficiency is described by the first term, $\varepsilon(d)$,

\footnotetext{
${ }^{4}$ Note that the cross section for the reaction $N+N \rightarrow d+\pi$ decreases rapidly with increasing energy.
} 
while the absorption loss (including annihilation) of $\bar{d}$ and $\bar{p}$ are assumed to be similar. This modelling assumes that the cross sections of annihilation in the detector material are the same for $\bar{d}$ and $\bar{p}$, since the inelastic nuclear cross section of $\bar{p}$ is much larger than that of $\bar{n}$ for the momentum region less than $0.4 \mathrm{GeV}$ [37]. The use of the geometrical model discussed in $[11,37]$ and the model in which the $\bar{p}$ and $\bar{n}$ inelastic absorption cross sections are added linearly $[4,37]$ to obtain the inelastic nuclear cross section of $\bar{d}$, reduces $\varepsilon(\bar{d})$ by $1 \%$ and $5 \%$, respectively.

\subsection{Efficiency of the $d E / d x$ cuts}

Another important contribution to the efficiency comes from the $d E / d x$ threshold cuts and the mass cuts. The inefficiency due to the $d E / d x$ requirements were estimated separately for positive and negative tracks using $\Lambda \rightarrow p \pi$ (+c.c.) decays. In this approach, protons were identified from the $\Lambda$ peak and then the proton $d E / d x$ selection efficiency was reconstructed as the ratio of the events without and with the $d E / d x$ requirement. These efficiencies were determined as a function of $p / M$. The efficiency for each $p_{T} / M$ bin was corrected by reweighting the $p / M$ distributions using ARIADnE. The average efficiency of the $d E / d x$ cuts for $d(\bar{d})$ is 0.7 for $p_{T} / M<0.5$. For larger momenta, the efficiency decreases due to the $d E / d x>2.5$ mips cut. The signal extraction is not possible for $p_{T} / M>0.7$ due to a very small efficiency. For the low-momentum region $p_{T} / M<0.5$, the efficiencies for negative tracks tend to be larger than for positive tracks. The $d E / d x$ efficiency for $p(\bar{p})$ is higher by $15 \%$ than that for $d(\bar{d})$.

Alternatively, the overall tracking and the $d E / d x$ efficiency was calculated using the ARIADNE MC model; consistent results with the approach discussed above were found.

\section{Systematic uncertainties}

The systematic uncertainties were evaluated by changing the selection and the analysis procedure. Only the largest contribution of each cut variation for the final invariant cross section is given below. The following sources of systematic uncertainties were studied:

- efficiency of the track reconstruction and selection. The systematic uncertainty on the tracking efficiency for $p, \bar{p}, d$ was $\pm 2 \%$. This systematic uncertainty was found after variations of the track-quality cuts. For $\bar{d}$, the systematic uncertainty, $\pm 5 \%$, includes both the effect of track-quality-cut variations and the reduction in $\varepsilon(\bar{d})$ when the linear model for the $\bar{d}$ absorption was used (see Section [7.1);

- efficiency due to the $d E / d x$ selection. This systematic uncertainty was estimated by varying the cut $d E / d x>2.5$ mips within the $d E / d x$ resolution and by using the MC simulation. This systematic uncertainty was $\pm 5 \%$. For the lowest $p_{T} / M$ bin, the uncertainty was $\pm 10 \%$; 
- variations in the particle yields associated with the signal extraction:

- the number of $d(\bar{d})$ were reconstructed using a Gaussian fit to the $D C A$ distribution with a first-order polynomial for the background description;

- the region used to determine the background for the side-band background subtraction was reduced to $1.5<|D C A|<3.5 \mathrm{~cm}$;

- the $D C A$ cut for the side-band background subtraction was varied within its resolution of $\pm 0.1 \mathrm{~cm}$;

- for the side-band background subtraction, the background shape was taken from the MC (without $d$ at the generator level);

- the cut on $\Delta Z$ was varied by $\pm 0.2 \mathrm{~cm}$;

These variations lowered the production yields by $5.0 \%$ for $p, 2.2 \%$ for $\bar{p}, 26.0 \%$ for $d$ and $6.1 \%$ for $\bar{d}$. The largest effect originates from the conservative treatment of the shape of the DCA background. The upper systematic error was below $1 \%$ for $p, \bar{p}$ and $\bar{d}$, and $11 \%$ for $d$.

- the background contribution under the $Z_{\mathrm{vtx}}$ peak for $d$ events was assumed to be due to beam-gas interactions and, therefore, it was subtracted $(-4 \%$ contribution for $p, \bar{p}$, $\bar{d}$ and $-17 \%$ contribution for $d)$;

- the correction for $\Lambda$ decays applied for the $p(\bar{p})$ sample was changed by $\pm 10 \%$ (see Section 9.1). The size of this uncertainty, which is similar to that in other publications $[4,9]$, was determined by the uncertainty on the strangeness suppression factor in the ARIADNE model;

- variations of the DIS-selection cuts. The cut on the energy of the scattered electron was increased to $10 \mathrm{GeV}$, and the lower cut on the $\delta$ distribution was tightened to $40 \mathrm{GeV}$. The cut on $Z_{\mathrm{vtx}}$ was varied by $\pm 5 \mathrm{~cm}$. The cut on the number of primary tracks was increased from three to four. These variations led to changes of ${ }_{-4.1}^{+3.3 \%}$ for $p,{ }_{-4.4}^{+3.6 \%}$ for $\bar{p},{ }_{-8.5}^{+3.7 \%}$ for $d$ and ${ }_{-13.3}^{+5.7} \%$ for $\bar{d}$. Variations of the cuts on $y_{e}$ and $y_{\mathrm{JB}}$ distributions showed a negligible effect.

The overall systematic uncertainty was determined by adding the above uncertainties in quadrature. The largest experimental uncertainty was due to the uncertainties on the tracking efficiency and the signal extraction.

\section{Results}

\subsection{Production cross sections and $B_{2}$}

For each particle type $i$, the invariant differential cross section can be calculated from the rapidity range $\Delta y$ and the transverse momentum $p_{T, i}$ of a corresponding particle through

$$
\frac{\gamma_{i}}{\sigma_{\text {tot }}} \frac{\mathrm{d}^{3} \sigma_{i}}{\mathrm{~d}\left(p_{i} / M_{i}\right)^{3}}=\frac{1}{N_{\text {DIS }}} \frac{1}{2 \pi\left(p_{T, i} / M_{i}\right) \Delta y} \frac{N_{i}}{\Delta\left(p_{T, i} / M_{i}\right)},
$$


where the subscript $i$ denotes a $p(\bar{p})$ or a $d(\bar{d}), N_{i}$ is the particle yield in each $p_{T, i} / M_{i}$ bin after the correction for the tracking efficiencies and the particle selection and $N_{\text {DIS }}=$ $2.59 \times 10^{7}$ is the number of DIS events used in the analysis. For the present measurement, $\Delta y=0.8$ and $\Delta\left(p_{T, i} / M_{i}\right)=0.1$ are the bin sizes. For comparisons with other experiments, the $p(\bar{p})$ rate was corrected for the decay products of $\Lambda$. A correction factor of 0.79 was estimated from the ARIADNE simulation which gives an adequate description of $K_{S}^{0}$ and $\Lambda$ production [38].

The invariant differential cross sections as a function of $p_{T} / M$ for $p(\bar{p})$ and $d(\bar{d})$ are shown in Fig. 7 and given in Tables 1 and 2. The $d(\bar{d})$ invariant cross section is smaller by approximately three orders of magnitude than that of $p(\bar{p})$. These cross sections were used to extract the coalescence parameter $B_{2}$ as discussed in Section 2 The parameter $B_{2}$ is shown in Fig. 8 and listed in Tables 3 and 4 . For $d, B_{2}$ tends to be higher than for $\bar{d}$, especially at low $p_{T} / M$. The value of $B_{2}$ for $\bar{d}$ is in agreement with the measurements in photoproduction [9], but larger than that observed in $e^{+} e^{-}$annihilation at the $Z$ resonance [4]. The measured $B_{2}$ is also significantly larger than that observed in heavyion collisions [11].

The events containing at least one $p(\bar{p})$ or $d(\bar{d})$ were analysed in the Breit frame [39]. The number of events with $p(\bar{p})$ in the current region of the Breit frame was about $2.5 \%$ of the total number of observed events with $p(\bar{p})$. In this region, neither $d$ nor $\bar{d}$ was found. Since the current region of the Breit frame is analogous to a single hemisphere of $e^{+} e^{-}$, the observation of $d(\bar{d})$ reported in this paper is not in contradiction with the low $\bar{d}$ rate observed in $e^{+} e^{-}[2-4]$.

\subsection{Production ratios}

The detector-corrected $d / p$ and $\bar{d} / \bar{p}$ ratios as a function of $p_{T} / M$ are shown in Fig. 9(a) and listed in Tables 3 and 4 . For the antiparticle ratio, there is a good agreement with the $\mathrm{H} 1$ published data for photoproduction [9], as well as with $p p$ data [8]. A similar $\bar{d} / \bar{p}$ ratio was also observed in hadronic $\Upsilon(1 S)$ and $\Upsilon(2 S)$ decays [2].

The $\bar{d} / d$ and $\bar{p} / p$ ratios as a function of $p_{T} / M$ are shown in Fig. 9(b) and listed in Table 5 , The $\bar{p} / p$ ratio is consistent with unity, as expected from hadronisation of quark and gluon jets. The dominant uncertainty on the ratio is due to systematic effects associated with the track selection and reconstruction.

The production rate of $d$ is higher than that of $\bar{d}$, especially at low $p_{T}$. Under the assumption that secondary interactions do not produce an enhancement at $D C A=0$ for the $d$ case, the result would indicate that the relation between $\bar{d} / d$ and $(\bar{p} / p)^{2}$ expected from the coalescence model does not hold in the central fragmentation region of ep DIS collisions.

For collisions involving incoming baryon beams, there are several models $[40,41]$ that predict baryon-antibaryon production asymmetry in the central rapidity region. A $p-\bar{p}$ 
asymmetry in proton-induced reactions is predicted to be as high as 7\% [41]. Given the experimental uncertainty, this measurement is not sensitive to the expected small $p-\bar{p}$ asymmetry.

In heavy-ion collisions, the $\bar{d}$ to $d$ production ratio is expected to be smaller than unity [42]. A recent measurement at RHIC [11] indicated a lower production rate of $\bar{d}$ compared to that of $d$. The average value of the ratio $\bar{d} / d=0.47 \pm 0.03$ was compatible with the square of the $\bar{p} / p=0.73 \pm 0.01$ ratio. Assuming the same size of the production volume for baryons and antibaryons, this RHIC result is consistent with the coalescence model. A similar conclusion was obtained earlier in fixed-target $p p$ [8] and $p A[7]$ experiments. For $e^{+} e^{-}$collisions, the $d$ yield is compatible with that of $\bar{d}$ within the large uncertainties $[4,5]$.

\section{Summary}

The first observation of $d(\bar{d})$ in $e p$ collisions in the DIS regime at HERA is presented. The production rate of $d(\bar{d})$ is smaller than that for $p(\bar{p})$ by three orders of magnitude, which is in broad agreement with other experiments.

The production of $d(\bar{d})$ was studied in terms of the coalescence model. The coalescence parameter is in agreement with the measurements in photoproduction at HERA. However, it is larger than that measured in $e^{+} e^{-}$annihilation at the $Z$ resonance.

The production rate of $p$ is consistent with that of $\bar{p}$ in the kinematic range $0.3<p_{T} / M<$ 0.7. Due to significant uncertainties, it is not possible to test models that predict a small baryon-antibaryon asymmetry in the central fragmentation region.

For the same kinematic region, the production rate of $d$ is higher than that for $\bar{d}$. If the observed $d$ are solely attributed to deuterons produced in primary ep collisions, the results would indicate that the coalescence model with the same source volume for $d$ and $\bar{d}$ cannot fully explain the production of $d(\bar{d})$ in DIS.

\section{Acknowledgements}

We thank the DESY Directorate for their strong support and encouragement. The remarkable achievements of the HERA machine group were essential for the successful completion of this work and are greatly appreciated. We are grateful for the support of the DESY computing and network services. The design, construction and installation of the ZEUS detector have been made possible owing to the ingenuity and effort of many people from DESY and home institutes who are not listed as authors. We thank Prof. D. Heinz and Prof. T. Sloan for the useful discussion of this topic. 


\section{References}

[1] T. Massam et al., Nuovo Cimento 39, 10 (1965).

[2] ARGUS Coll., H. Albrecht et al., Phys. Lett. B 157, 326 (1985);

ARGUS Coll., H. Albrecht et al., Phys. Lett. B 236, 102 (1990).

[3] OPAL Coll., R. Akers, et al., Z. Phys. C 67, 203 (1995).

[4] ALEPH Coll., S. Schael et al., Phys. Lett. B 639, 16 (2006).

[5] CLEO Coll., D.M. Asner et al., Phys. Rev. D 75, 012009 (2007).

[6] IHEP-CERN Coll., F. Binon et al., Phys. Lett. B 30, 510 (1969);

Yu.M. Antipov et al., Phys. Lett. B 34, 164 (1971).

[7] J.W. Cronin et al., Phys. Rev. D 11, 3105 (1975).

[8] B. Alper et al., Phys. Lett. B 46, 265 (1973);

British-Scandinavian Coll., W.M. Gibson et al., Nuovo Cim. Lett. 21, 189 (1978);

V.V. Abramov, Sov. J. Nucl. Phys. 45, 845 (1987).

[9] H1 Coll., A. Aktas et al., Eur. Phys. J. C 36, 413 (2004).

[10] M. Aoki et al., Phys. Rev. Lett. 69, 2345 (1992);

NA52 (NEWMASS) Coll., G. Appelquist et al., Phys. Lett. B 376, 245 (1996);

STAR Coll., C. Alper et al., Phys. Rev. Lett. 87, 262301 (2001);

E802 Coll., L. Ahle et al., Phys. Rev. C 57, 1416 (1998);

NA44 Coll., I.G. Bearden et al., Nucl. Phys. A 661, 387 (1999);

NA44 Coll., I.G. Bearden et al., Eur. Phys. J. C 23, 237 (2002).

[11] PHENIX Coll., S.S. Adler et al., Phys. Rev. Lett. 94, 122302 (2005).

[12] S.T. Butler and C.A. Pearson, Phys. Rev. 129, 836 (1963).

[13] ZEUS Coll., U. Holm (ed.), The ZEUS Detector. Status Report (unpublished),

DESY (1993), available on http://www-zeus.desy.de/bluebook/bluebook.html.

[14] N. Harnew et al., Nucl. Inst. Meth. A 279, 290 (1989);

B. Foster et al., Nucl. Phys. Proc. Suppl. B 32, 181 (1993);

B. Foster et al., Nucl. Inst. Meth. A 338, 254 (1994).

[15] ZEUS Coll., J. Breitweg et al., Phys. Lett. B 481, 213 (2000);

ZEUS Coll., J. Breitweg et al., Eur. Phys. J. C 18, 625 (2001);

D. Bartsch. Ph.D. Thesis (unpublished), Universität Bonn, Bonn, Germany, 2007.

[16] M. Derrick et al., Nucl. Inst. Meth. A 309, 77 (1991);

A. Andresen et al., Nucl. Inst. Meth. A 309, 101 (1991);

A. Caldwell et al., Nucl. Inst. Meth. A 321, 356 (1992);

A. Bernstein et al., Nucl. Inst. Meth. A 336, 23 (1993).

[17] A. Bamberger et al., Nucl. Inst. Meth. A 382, 419 (1996);

S. Magill and S. Chekanov, Proceedings of the IX Int. Conference on Calorimetry 
(Annecy, Oct 9-14, 2000), B. Aubert et al. (ed.), p. 625. Frascati Physics Series 21, Annecy, France (2001).

[18] A. Bamberger et al., Nucl. Inst. Meth. A 401, 63 (1997).

[19] J. Andruszków et al., Preprint DESY-92-066, DESY, 1992;

ZEUS Coll., M. Derrick et al., Z. Phys. C 63, 391 (1994);

J. Andruszków et al., Acta Phys. Pol. B 32, 2025 (2001).

[20] L. Lönnblad, Comp. Phys. Comm. 71, 15 (1992).

[21] B. Andersson et al., Phys. Rep. 97, 31 (1983).

[22] M. Bengtsson and T. Sjöstrand, Comp. Phys. Comm. 46, 43 (1987).

[23] T. Sjöstrand, Comp. Phys. Comm. 82, 74 (1994).

[24] T. Sjöstrand et al., Comp. Phys. Comm. 135, 238 (2001).

[25] R. Brun et al., GEANT3, Technical Report CERN-DD/EE/84-1, CERN, 1987.

[26] H. Fesefeldt, The simulation of hadronic showers: physics and applications (unpublished). PITHA-85-02.

[27] ZEUS Coll., S. Chekanov et al., Phys. Lett. B 591, 7 (2004).

[28] H. Abramowicz, A. Caldwell and R. Sinkus, Nucl. Inst. Meth. A 365, 508 (1995).

[29] F. Jacquet and A. Blondel, Proceedings of the Study for an ep Facility for Europe, U. Amaldi (ed.), p. 391. Hamburg, Germany (1979). Also in preprint DESY 79/48.

[30] Particle Data Group, W.-M. Yao et al., J. Phys. G33, 1 (2006).

[31] N. Blasi et al., Nucl. Phys. A 624, 433 (1997).

[32] J. Franz et al., Nucl. Phys. A 472, 733 (1987).

[33] G.R. Smith et al., Phys. Rev. C 30, 593 (1984).

[34] K. Kikuchi, Prog. of Theor. Phys. 18, 503 (1957).

[35] P.G. Roos et al., Nucl. Phys. A 255, 187 (1975).

[36] B. Fagerstrom et al., Physica Scripta 13, 10 (1976).

[37] A.A. Moiseev and J.F. Ormes, Astroparticle Physics 6, 379 (1997).

[38] ZEUS Coll., S. Chekanov et al., 2006. DESY-06-226 (Eur. Phys. J. C, in press.).

[39] R.P. Feynman, Photon-Hadron Interactions. Benjamin, New York, 1972;

K.H. Streng, T.F. Walsh and P.M. Zerwas, Z. Phys. C 2, 237 (1979).

[40] G.T. Garvey, B.Z. Kopeliovich and B. Povh, Comments Mod. Phys. A 2, 47 (2001);

S. Chekanov, Eur. Phys. J. C 44, 367 (2005);

F. Bopp and Yu.M. Shabelski, Phys. Atom. Nucl. 68, 2093 (2005);

F. Bopp and Yu.M. Shabelski, Eur. Phys. J. A 28, 237 (2006).

[41] B. Kopeliovich and B. Povh, Z. Phys. C 75, 693 (1997);

B. Kopeliovich and B. Povh, Phys. Lett. B 446, 321 (1999). 
[42] S. Leupold and U.W. Heinz, Phys. Rev. C50, 1110 (1994). 


\begin{tabular}{|c|c|c|}
\hline$p_{T} / M$ & $\left(\gamma_{p} / \sigma_{\text {tot }}\right) \mathrm{d}^{3} \sigma_{p} / \mathrm{d}\left(p_{p} / M_{p}\right)^{3}\left(\times 10^{-2}\right)$ & $\left(\gamma_{d} / \sigma_{\text {tot }}\right) \mathrm{d}^{3} \sigma_{d} / \mathrm{d}\left(p_{d} / M_{d}\right)^{3}\left(\times 10^{-5}\right)$ \\
\hline \hline $0.3-0.4$ & $1.33 \pm 0.01_{-0.21}^{+0.19}$ & $3.29 \pm 0.43_{-1.24}^{+0.50}$ \\
\hline $0.4-0.5$ & $1.34 \pm 0.01_{-0.18}^{+0.16}$ & $1.37 \pm 0.26_{-0.51}^{+0.17}$ \\
\hline $0.5-0.6$ & $0.88 \pm 0.01_{-0.12}^{+0.10}$ & $1.16 \pm 0.28_{-0.42}^{+0.14}$ \\
\hline $0.6-0.7$ & $0.38 \pm 0.01_{-0.05}^{+0.04}$ & - \\
\hline
\end{tabular}

Table 1: The measured invariant cross sections for the production of $p$ and $d$ in $D I S$ as a function of $p_{T} / M$. The statistical and systematic uncertainties are also listed.

\begin{tabular}{|c|c|c|}
\hline$p_{T} / M$ & $\left(\gamma_{\bar{p}} / \sigma_{\text {tot }}\right) \mathrm{d}^{3} \sigma_{\bar{p}} / \mathrm{d}\left(p_{\bar{p}} / M_{\bar{p}}\right)^{3}\left(\times 10^{-2}\right)$ & $\left(\gamma_{\bar{d}} / \sigma_{\text {tot }}\right) \mathrm{d}^{3} \sigma_{\bar{d}} / \mathrm{d}\left(p_{\bar{d}} / M_{\bar{d}}\right)^{3}\left(\times 10^{-5}\right)$ \\
\hline \hline $0.3-0.4$ & $1.59 \pm 0.01_{-0.19}^{+0.16}$ & $0.77 \pm 0.15_{-0.14}^{+0.09}$ \\
\hline $0.4-0.5$ & $1.21 \pm 0.01_{-0.09}^{+0.07}$ & $0.45 \pm 0.11_{-0.07}^{+0.03}$ \\
\hline $0.5-0.6$ & $0.86 \pm 0.01_{-0.07}^{+0.05}$ & $0.60 \pm 0.19_{-0.09}^{+0.05}$ \\
\hline $0.6-0.7$ & $0.35 \pm 0.01_{-0.03}^{+0.02}$ & - \\
\hline
\end{tabular}

Table 2: $\quad$ The measured invariant cross sections for the production of $\bar{p}$ and $\bar{d}$ in DIS as a function of $p_{T} / M$. The statistical and systematic uncertainties are also listed.

\begin{tabular}{|c|c|c|}
\hline$p_{T} / M$ & $R(d / p)\left(\times 10^{-3}\right)$ & $B_{2}(d)\left(10^{-2} \mathrm{GeV}^{2}\right)$ \\
\hline \hline $0.3-0.4$ & $2.48 \pm 0.33_{-1.00}^{+0.55}$ & $4.11 \pm 0.54_{-1.97}^{+1.47}$ \\
\hline $0.4-0.5$ & $1.02 \pm 0.19_{-0.40}^{+0.19}$ & $1.68 \pm 0.32_{-0.74}^{+0.50}$ \\
\hline $0.5-0.6$ & $1.32 \pm 0.32_{-0.51}^{+0.24}$ & $3.31 \pm 0.80_{-1.45}^{+0.99}$ \\
\hline $0.6-0.7$ & - & \\
\hline \hline $0.3-0.7$ & $1.88 \pm 0.20_{-0.75}^{+0.40}$ & $3.32 \pm 0.34_{-1.55}^{+1.13}$ \\
\hline
\end{tabular}

Table 3: $\quad$ The measured d-to-p production ratio and the parameter $B_{2}$ for $d$ as a function of $p_{T} / M$. The last row of the table shows the data in the full measured phase space. The statistical and systematic uncertainties are also listed.

\begin{tabular}{|c|c|c|}
\hline$p_{T} / M$ & $R(\bar{d} / \bar{p})\left(\times 10^{-3}\right)$ & $B_{2}(\bar{d})\left(10^{-2} \mathrm{GeV}^{2}\right)$ \\
\hline \hline $0.3-0.4$ & $0.48 \pm 0.09_{-0.10}^{+0.08}$ & $0.67 \pm 0.13_{-0.19}^{+0.18}$ \\
\hline $0.4-0.5$ & $0.37 \pm 0.09_{-0.06}^{+0.04}$ & $0.67 \pm 0.17_{-0.13}^{+0.12}$ \\
\hline $0.5-0.6$ & $0.70 \pm 0.22_{-0.12}^{+0.08}$ & $1.80 \pm 0.57_{-0.36}^{+0.31}$ \\
\hline $0.6-0.7$ & - & - \\
\hline \hline $0.3-0.7$ & $0.49 \pm 0.07_{-0.09}^{+0.07}$ & $0.89 \pm 0.14_{-0.20}^{+0.19}$ \\
\hline
\end{tabular}

Table 4: $\quad$ The measured $\bar{d}-$ to- $\bar{p}$ production ratio and the parameter $B_{2}$ for $\bar{d}$ as a function of $p_{T} / M$. The last row of the table shows the data in the full measured phase space. The statistical and systematic uncertainties are also listed. 


\begin{tabular}{|c|c|c|}
\hline$p_{T} / M$ & $R(\bar{p} / p)$ & $R(\bar{d} / d)$ \\
\hline \hline $0.3-0.4$ & $1.19 \pm 0.01_{-0.19}^{+0.20}$ & $0.23 \pm 0.05_{-0.05}^{+0.09}$ \\
\hline $0.4-0.5$ & $0.90 \pm 0.01_{-0.09}^{+0.10}$ & $0.33 \pm 0.10_{-0.07}^{+0.12}$ \\
\hline $0.5-0.6$ & $0.97 \pm 0.01_{-0.10}^{+0.11}$ & $0.52 \pm 0.21_{-0.10}^{+0.19}$ \\
\hline $0.6-0.7$ & $0.92 \pm 0.03_{-0.09}^{+0.10}$ & - \\
\hline \hline $0.3-0.7$ & $1.05 \pm 0.01_{-0.14}^{+0.15}$ & $0.31 \pm 0.05_{-0.06}^{+0.11}$ \\
\hline
\end{tabular}

Table 5: $\quad$ The measured $\bar{p}$-to-p and $\bar{d}$-to-d production ratios as a function of $p_{T} / M$. The last row of the table shows the data in the full measured phase space. The statistical and systematic uncertainties are also listed. 

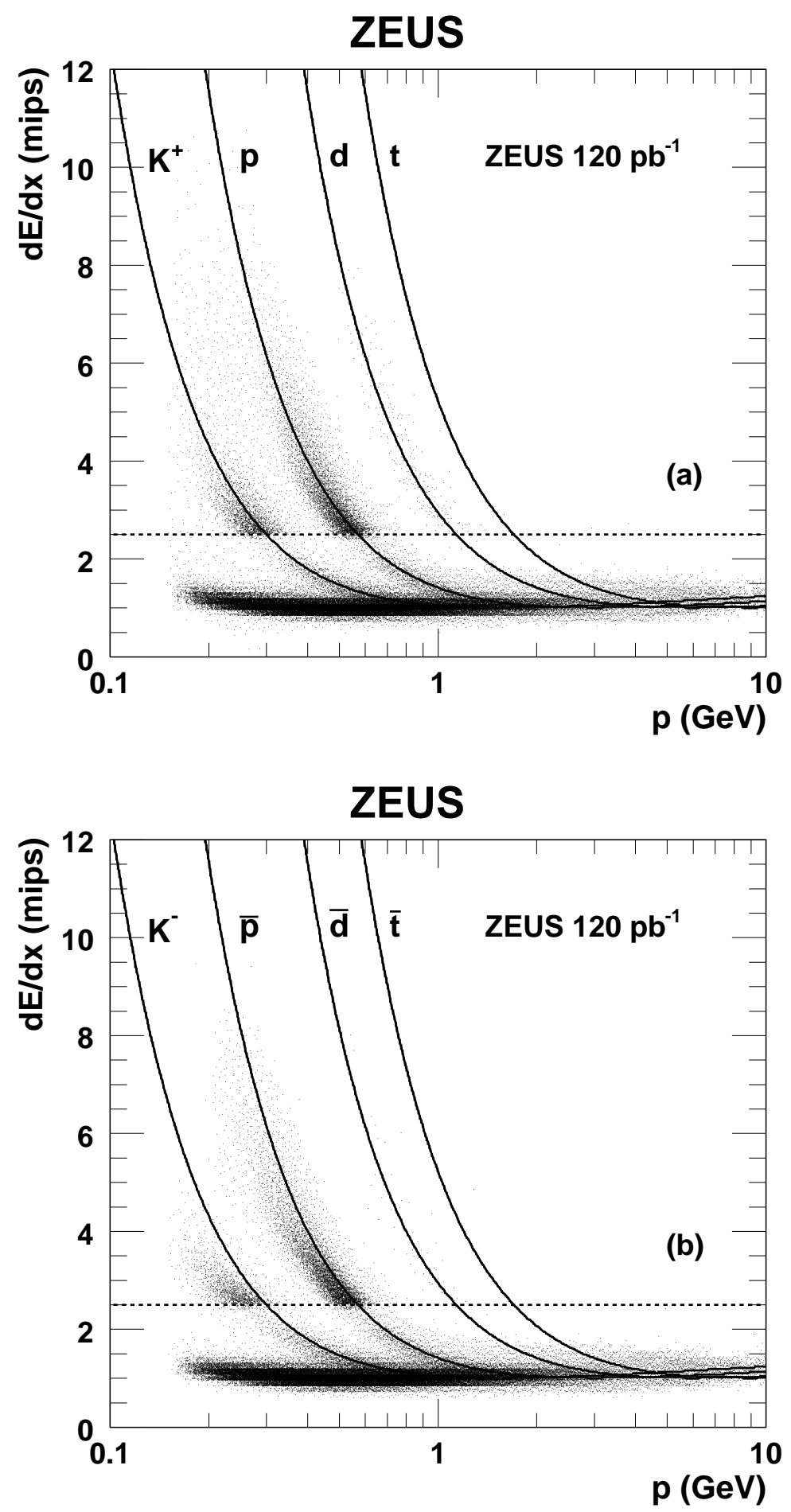

Figure 1: The $d E / d x$ distributions as a function of the track momentum for (a) positive and (b) negative tracks. The DIS events were accepted by requiring at least one track with $d E / d x>2.5$ mips (denoted by the dashed lines), $|\Delta Z|<1 \mathrm{~cm}$ and $|D C A|<0.5 \mathrm{~cm}$. The lines show the most-probable energy loss calculated using the Bethe-Bloch formula for different particle species. 
ZEUS
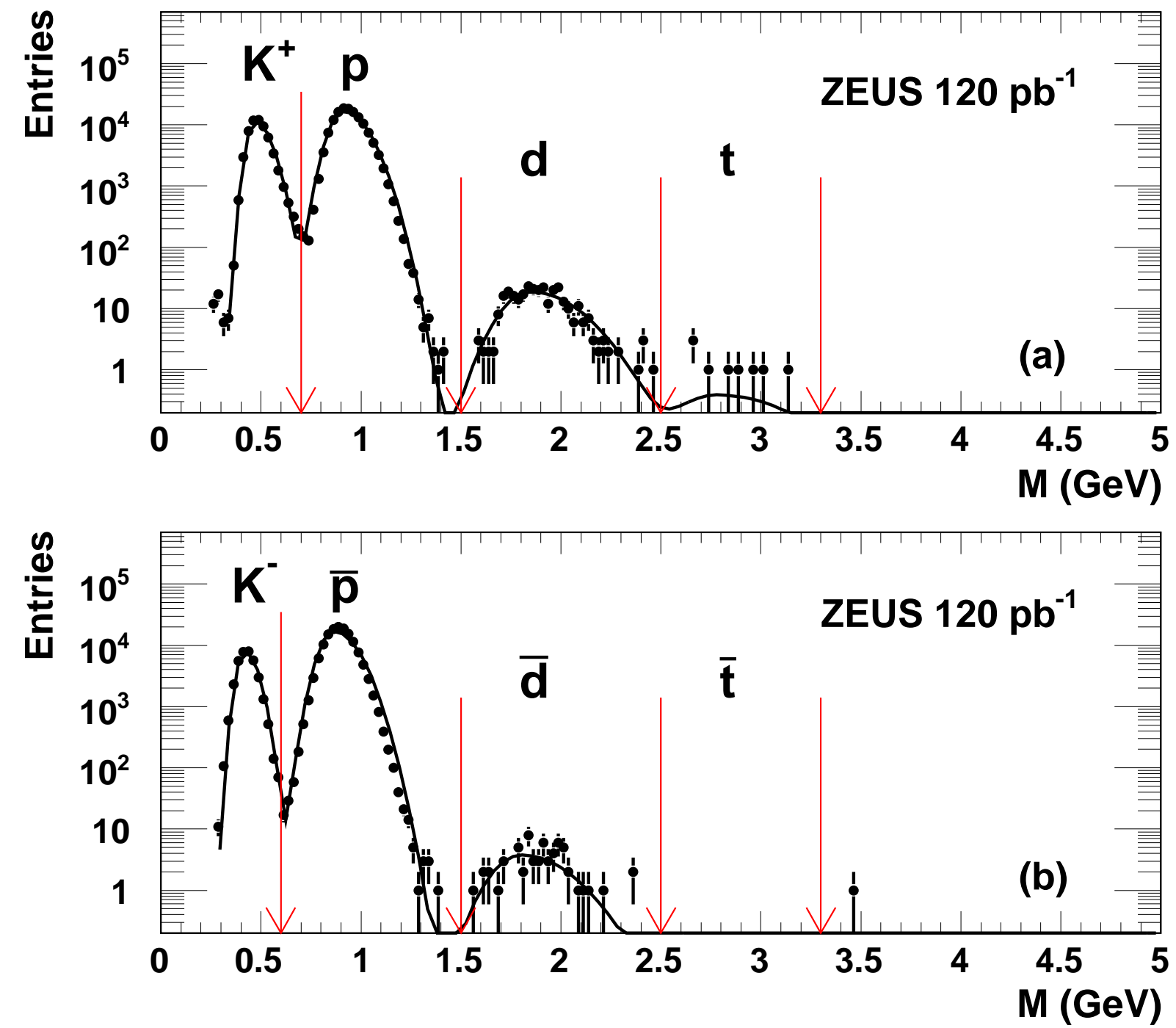

Figure 2: The mass spectra for (a) positive and (b) negative particles. Tracks are selected as for Figure [1] The mass distribution was calculated from the track momenta and the $d E / d x$. The arrows indicate the cuts applied for the selection of candidates. 


\section{ZEUS}
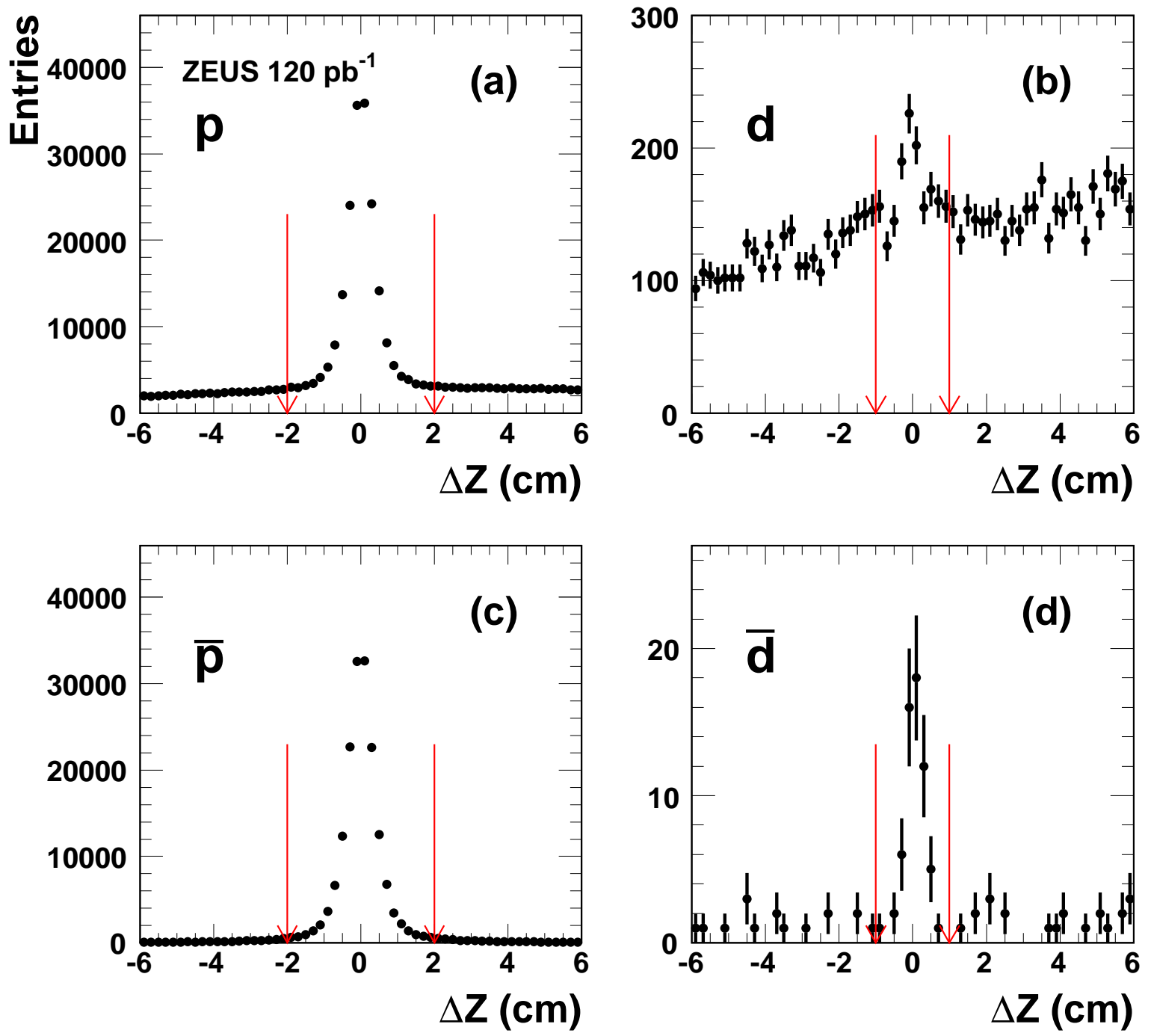

Figure 3: The distributions of $\Delta Z$, the distance of the $Z$-component of the track helix to $Z_{\mathrm{vtx}}$ for: (a)-(b) particles and (c)-(d) antiparticles, as indicated in the figure. The $p, \bar{p}, d$ and $\bar{d}$ candidates were identified using the $d E / d x$ mass cuts (see text). The arrows indicate the applied cuts. 


\section{ZEUS}
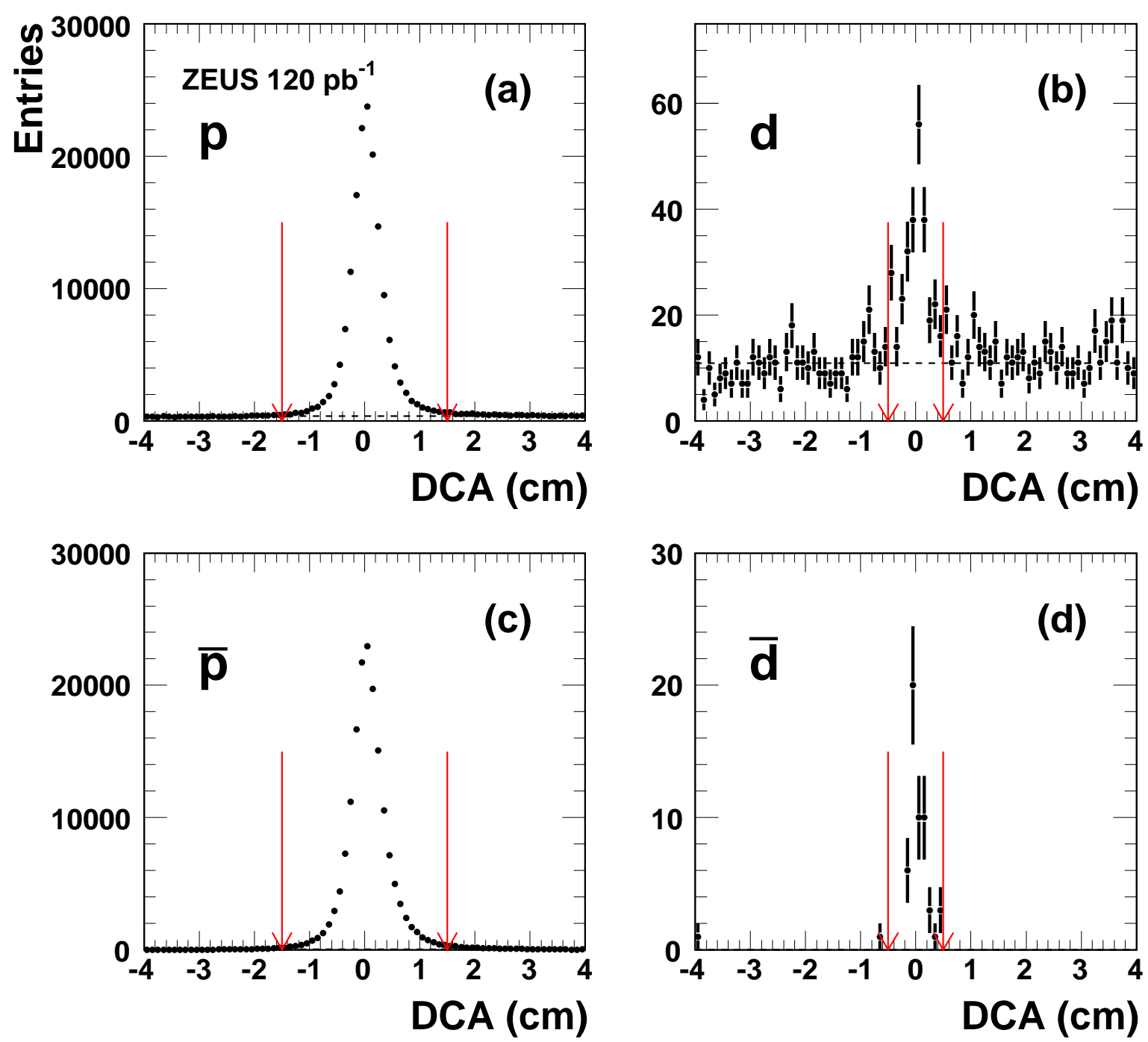

Figure 4: The distributions of the distance of closest approach, DCA, for: (a)(b) particles and (c)-(d) antiparticles. The DCA are shown after the cut $|\Delta Z|<$ $2(1) \mathrm{cm}$ as discussed in the text. The arrows indicate the signal region for the sideband background subtraction. The dashed lines show the fitted background level. 


\section{ZEUS}
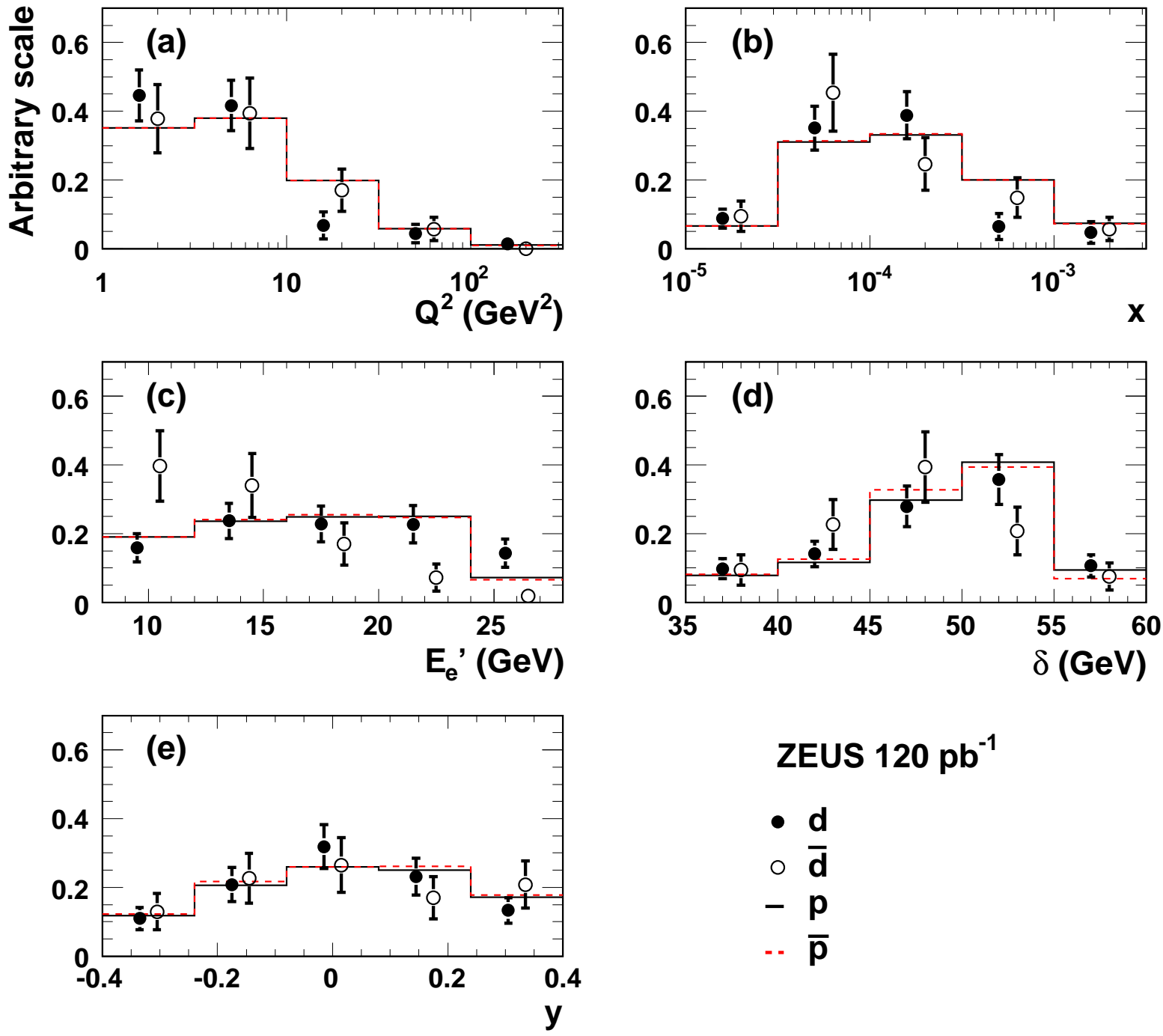

ZEUS $120 \mathrm{pb}^{-1}$

- $d$

$\circ \bar{d}$

$-p$

-. $\overline{\mathbf{p}}$

Figure 5: The distributions of the number of events with at least one $d(\bar{d})$ and $p(\bar{p})$ candidate normalised to unity as a function of: (a)-(d) DIS kinematic variables and (e) rapidity $y$. The points for $d$ and $\bar{d}$ are slightly shifted horizontally for clarity. 
ZEUS
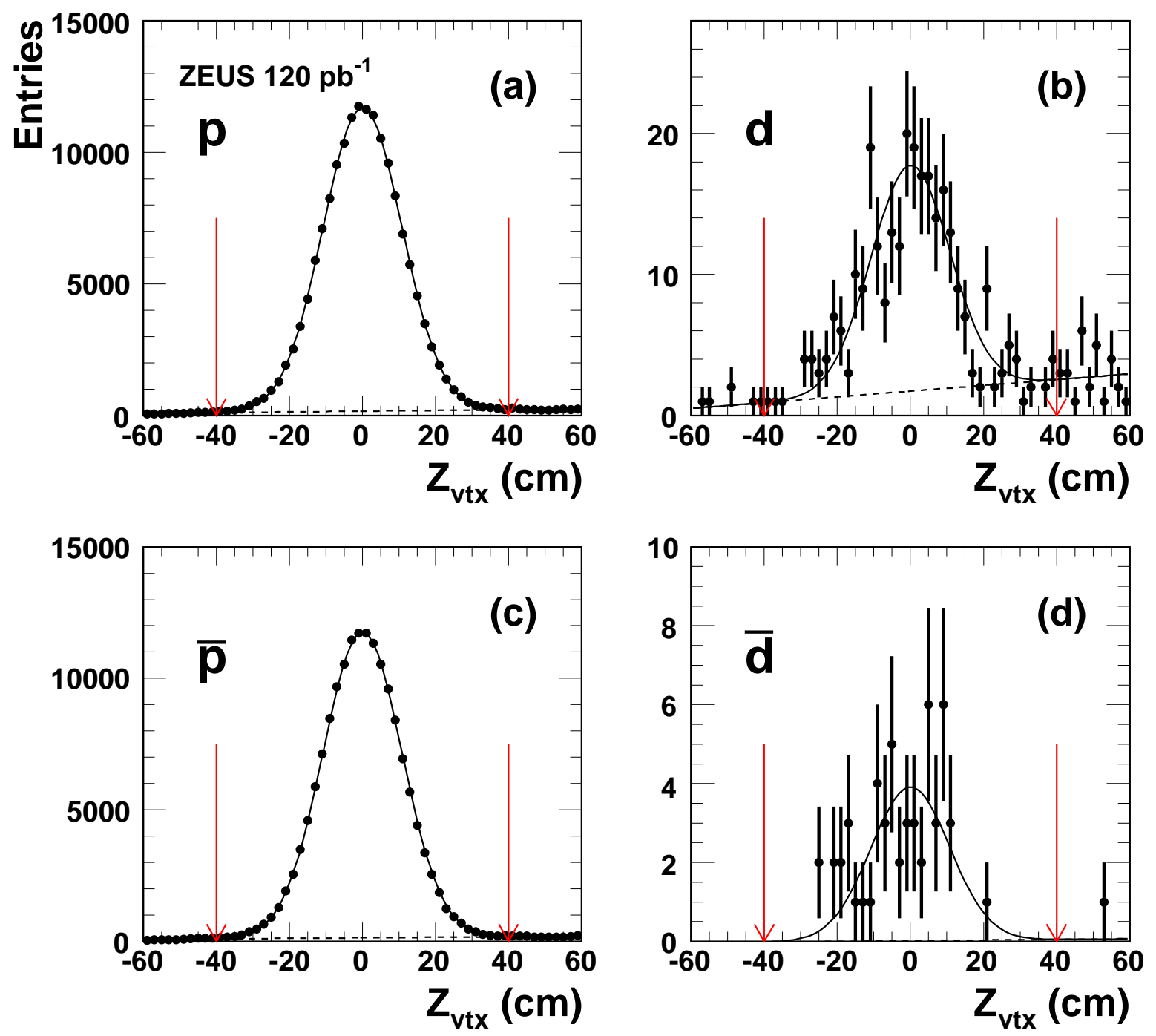

Figure 6: The $Z_{\mathrm{vtx}}$ distributions for: (a)-(b) particles and (c)-(d) antiparticles, as indicated in the figure. The solid lines show the fit using a Gaussian distribution with a first-order polynomial function for the background description. The dashed line shows the fitted background. The arrows indicate the cuts applied for the final selection. 


\section{ZEUS}

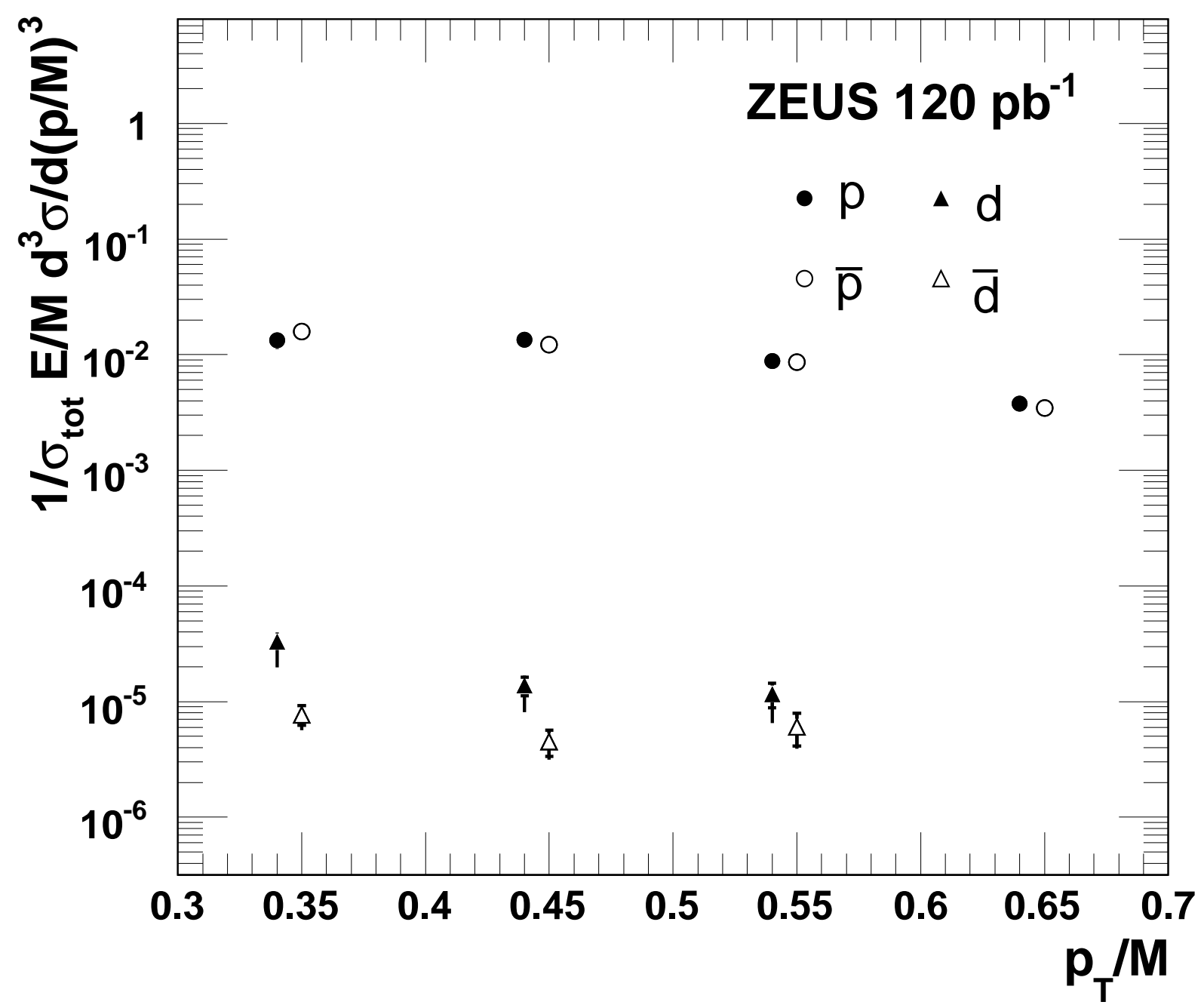

Figure 7: The invariant differential cross sections for $p(\bar{p})$ and $d(\bar{d})$ produced in $D I S$ ep collisions as a function of $p_{T} / M$. The inner error bars show the statistical uncertainties, the outer ones show statistical and systematic uncertainties added in quadrature. For clarity, the points for particles and antiparticles are slightly shifted horizontally with respect to the corresponding $p_{T} / M$. 


\section{ZEUS}

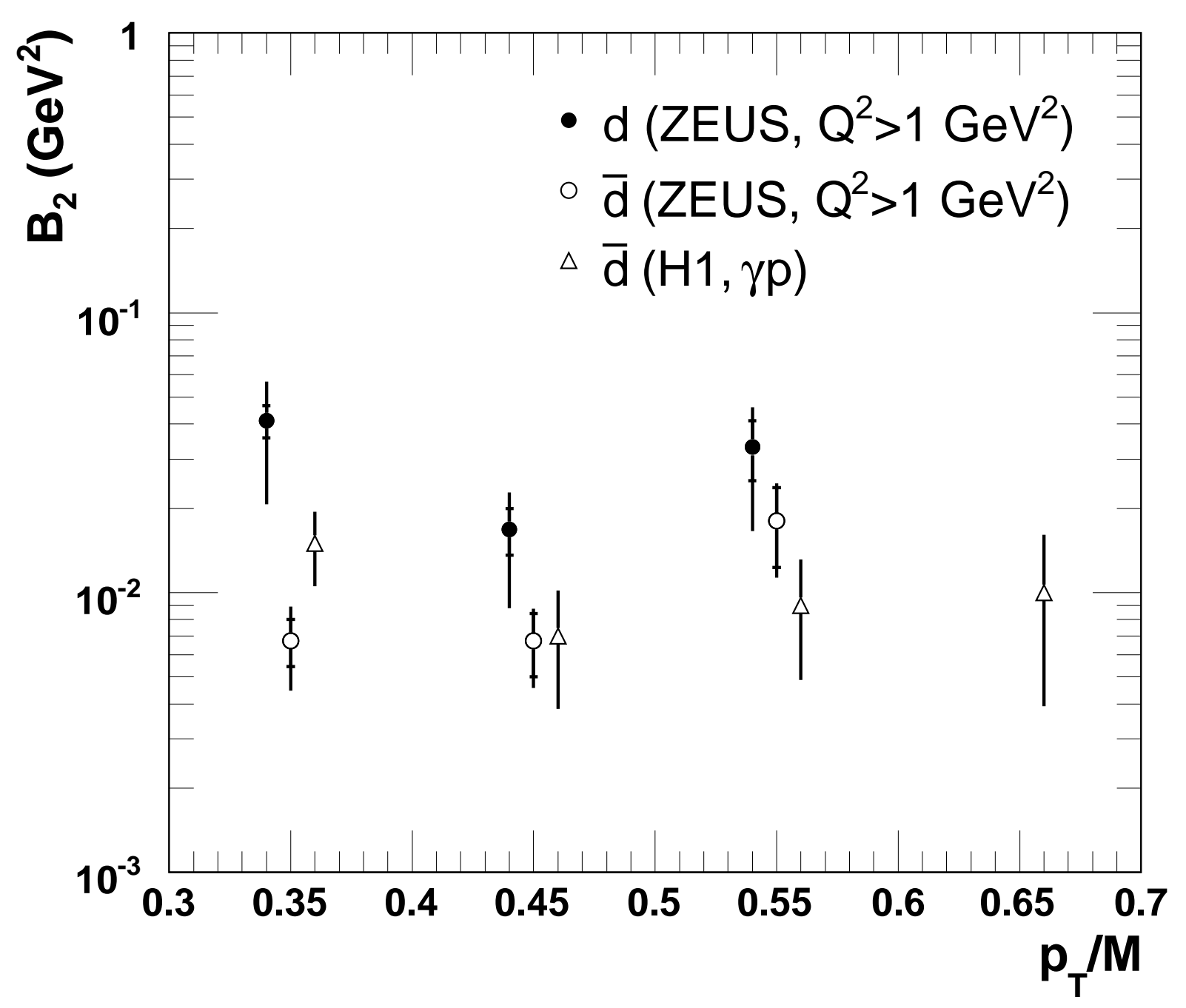

Figure 8: The $p_{T} / M$ dependence of the parameter $B_{2}$ for $d$ and $\bar{d}$ produced in DIS ep collisions and in photoproduction [9]. The inner error bars show the statistical uncertainties, the outer ones show statistical and systematic uncertainties added in quadrature. For clarity, the points for particles and antiparticles are slightly shifted horizontally with respect to the corresponding $p_{T} / M$. 


\section{ZEUS}
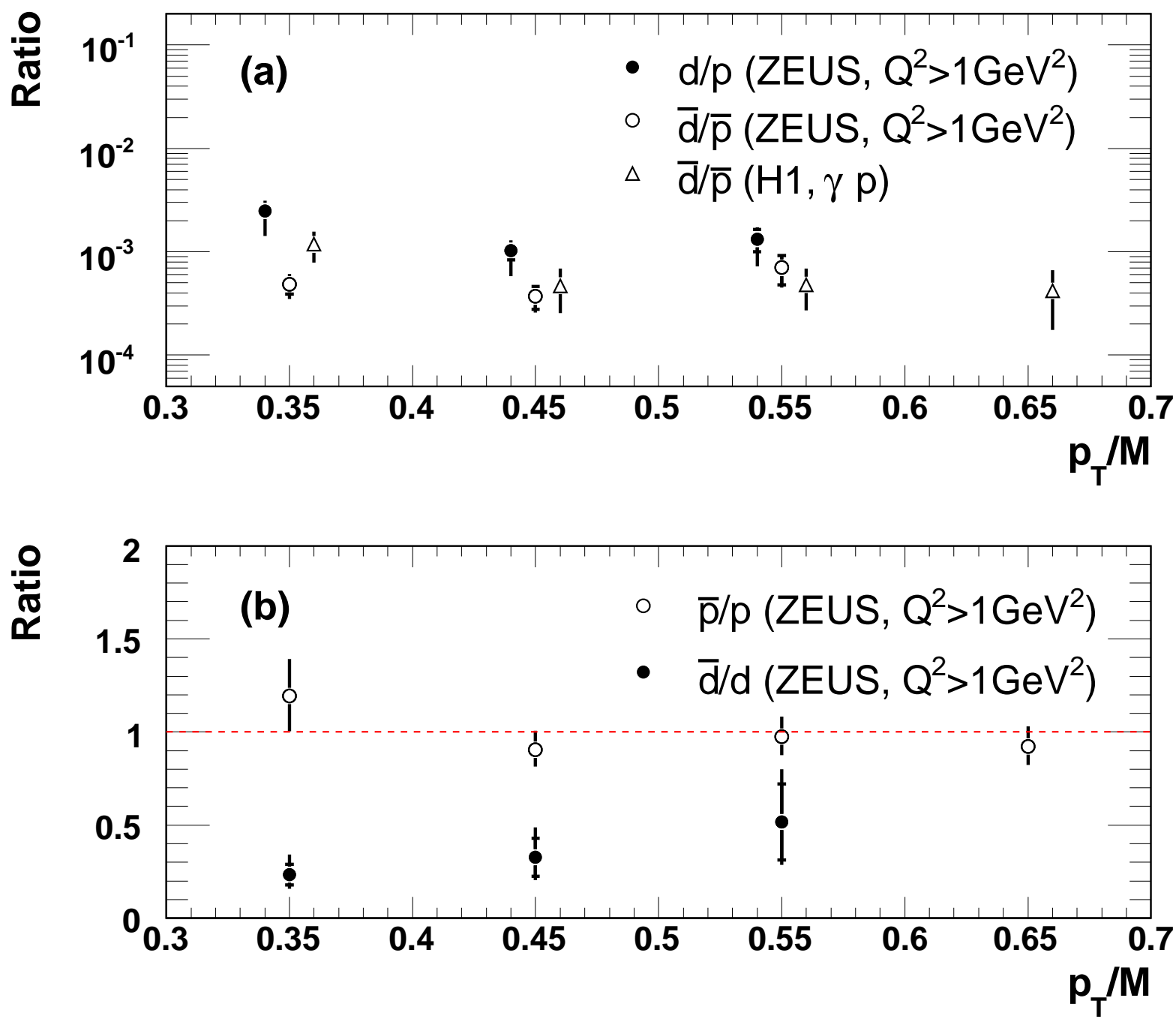

Figure 9: (a) $d / p$ and $\bar{d} / \bar{p}$ production ratios as a function of $p_{T} / M$ compared to the $H 1$ photoproduction results [9]. (b) the $\bar{d} / d$ and $\bar{p} / p$ production ratios as a function of $p_{T} / M$. The inner error bars show the statistical uncertainties, the outer ones show statistical and systematic uncertainties added in quadrature. The points in (a) are slightly shifted horizontally for clarity. 